\title{
Clinical course and management of postoperative methicillin-resistant Staphylococcus aureus keratitis in immunocompromised patients: two case reports
}

\author{
This article was published in the following Dove Press journal: \\ Clinical Ophthalmology \\ 19 December 2011 \\ Number of times this article has been viewed
}

\section{Timothy Y Chou' \\ Sujata P Prabhu ${ }^{2}$ \\ 'Department of Ophthalmology, State University of New York Stony Brook, Stony Brook, NY, ${ }^{2}$ Shiley Eye Center, University of California San Diego, La Jolla, CA, USA}

\begin{abstract}
We describe the clinical course and successful treatment of two cases of methicillin-resistant Staphylococcus aureus (MRSA) keratitis. In case 1, MRSA keratitis occurred 5 days after cataract extraction, associated with endophthalmitis; in case 2, diagnosis was made 19 months after penetrating keratoplasty. Treatment in both cases consisted of topical fortified vancomycin and fortified bacitracin. A third topical antibiotic, polymyxin B-trimethoprim, was added to the therapeutic regimen in case 2, one month into the treatment. Oral doxycycline was prescribed to reduce collagenase activity and treat blepharitis. Mupirocin nasal ointment and skin antiseptics were used to decrease and eliminate potential MRSA colonization. Topical prednisolone acetate $1 \%$ was applied conservatively to mitigate inflammation in both cases. In case 2, topical cyclosporine A was also used for similar purposes. Keratitis may have worsened while on these immune-modulating drops, especially in case 2, and eradication of infection may have been slowed. Eventually both patients achieved full resolution of infection. Duration of keratitis was 3 and 1.5 months, respectively. Polyantimicrobial therapy is effective in eradicating MRSA-related postoperative keratitis. Topical fortified vancomycin and fortified bacitracin were used in both cases, with a third topical antibiotic, polymyxin B-trimethoprim, also required in case 2. Oral doxycycline, nasal mupirocin, and antiseptic soap may be useful adjuncts in management. Treatment time to achieve full resolution may be prolonged relative to other types of bacterial keratitis. Alterations in immune status may have lengthened the time of treatment. Our two patients were immune compromised and were also susceptible to endophthalmitis. It is possible that topical immune-modulating drops such as prednisolone acetate may potentiate MRSA infection, and if used, should be only done so with great caution.
\end{abstract}

Keywords: cornea, infection, methicillin-resistant Staphylococcus aureus, cataract, keratoplasty, endophthalmitis

\section{Introduction}

Reports of ocular methicillin-resistant Staphylococcus aureus (MRSA) infections have been increasing in recent years as the prevalence of this organism increases. ${ }^{1,2}$ Blomquist retrospectively examined all culture-positive cases of MRSA from a public health care system over a 4-year period, finding that $1.3 \%$ had an ocular source. ${ }^{2}$ In their analysis of MRSA isolates from the Proctor clinical laboratory database, Freidlin et al determined that $14.6 \%$ of their ocular infections had a diagnosis of keratitis. ${ }^{3}$ However, to date, there have been relatively few reports describing a precise treatment approach to MRSA-related keratitis. ${ }^{2-5}$ Herein, we report two cases of postoperative
Correspondence: Timothy $Y$ Chou SUNY Stony Brook, HSC L2, Room I52, Stony Brook, NY I I794, USA

$\mathrm{Tel}+$ I 63I 444 II09

Fax + I 63| 444 I543

Email timothy.chou@stonybrook.edu 
MRSA keratitis, one after cataract surgery and one after corneal transplant. The medical records of these two patients were retrospectively reviewed. Data extraction included age, gender, medical history and risk factors for infection, eye culture results, antimicrobial susceptibilities, and treatment regimen. The clinical course and our therapeutic regimen are detailed.

\section{Case I}

A 52-year-old male with multiple medical problems, including uncontrolled type 1 diabetes mellitus, chronic ischemic cardiomyopathy, and hypertension, as well as a number of emergency room and hospital admissions in the previous year, underwent uncomplicated cataract extraction of the right eye. On postoperative day 5, experiencing progressively worsening vision and pain, the patient was diagnosed with endophthalmitis. Concurrently, the patient was noted to have a $1.5 \times 1 \mathrm{~mm}$ corneal infiltrate in the surgical paracentesis site (Figure 1). Vitreous cultures grew MRSA, which showed additional resistance to ampicillinsulbactam, cefazolin, ciprofloxacin, clindamycin, levofloxacin, oxacillin, and penicillin. Topical fortified vancomycin $25 \mathrm{mg} / \mathrm{mL}$ and tobramycin $14 \mathrm{mg} / \mathrm{mL}$ drops were started on an hourly basis, while postoperative prednisolone acetate $1 \%$ was continued four times per day. The patient also received intravitreal injections of ceftazidime and vancomycin. Additional injections of vancomycin were required on two subsequent occasions when the endophthalmitis appeared to flare. By postoperative day 15 , the corneal infiltrate had increased in size to $2 \times 1.75 \mathrm{~mm}$, with a larger hypopyon. The tobramycin drops were discontinued, and replaced with fortified bacitracin $3000 \mathrm{U} / \mathrm{mL}$; vancomycin was increased in concentration to $50 \mathrm{mg} / \mathrm{mL}$. As adjunctive

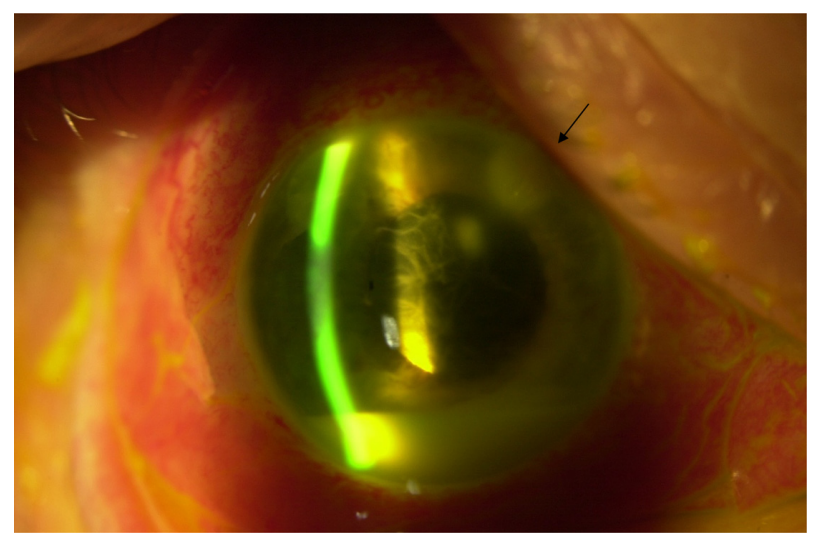

Figure I Slit lamp photograph on postoperative day 10 showing keratitis and endophthalmitis.

Note: Arrow points to infiltrate at paracentesis incision site. therapy, mupirocin calcium nasal ointment was prescribed twice a day for five days to decrease the potential for MRSA colonization, and doxycycline $100 \mathrm{mg}$ orally twice a day was added to treat blepharitis. The patient was instructed to use an ethyl alcohol instant hand sanitizer several times daily. He seemed to show slight improvement, but one month postoperatively the infiltrate remained active, and there was a small new hypopyon. Although there was no growth on repeat corneal cultures, the patient nevertheless was started on a third topical antibiotic, polymyxin B-trimethoprim, at an initial dosing interval of every two hours. Prednisolone acetate was decreased to twice daily. On this regimen the infiltrate slowly improved and ultimately resolved. The duration of the entire keratitis treatment totaled 3 months. The patient's blood sugar was not well controlled during the period of infection, and was often above $140 \mathrm{mg} / \mathrm{dL}$ and even $200 \mathrm{mg} / \mathrm{dL}$ on one occasion.

\section{Case 2}

A 75-year-old female with multiple myeloma underwent cataract extraction in the right eye, complicated by a postoperative corneal ulcer and endophthalmitis. No etiologic agent was identified at the time, but the condition resolved with broad spectrum antibiotic therapy. Subsequently she underwent penetrating keratoplasty in the right eye for postinfectious corneal scarring. Nineteen months later, the patient was diagnosed with a corneal rejection. She was started on hourly topical prednisolone acetate 1\%. Five days later, experiencing increased pain, she was found to have a suture abscess (Figure 2). The suture was removed, and the patient was started on topical levofloxacin 1.5\% every 30 minutes. Prednisolone acetate $1 \%$ was reduced to once per day. Hourly topical fortified vancomycin $50 \mathrm{mg} /$ $\mathrm{mL}$ was added when cultures were positive for $S$. aureus. Several days later, when substantial corneal thinning was observed, prednisolone acetate was discontinued entirely, cyanoacrylate glue applied, and oral doxycycline $50 \mathrm{mg}$ twice daily prescribed. After antimicrobial susceptibility testing revealed resistance to methicillin as well as to levofloxacin, erythromycin, and oxacillin, the fluoroquinolone drops were replaced with topical fortified bacitracin $3000 \mathrm{U} / \mathrm{mL}$. Despite initial improvement, after two weeks of treatment, the infiltrate began to appear a little denser as topical antibiotics were gradually tapered. The cornea was recultured and this time coagulase-negative Staphylococcus capitus was grown. Vancomycin and bacitracin were increased to greater frequency, resulting in lessening of the infiltrate again. At week 3 of treatment, topical cyclosporine 


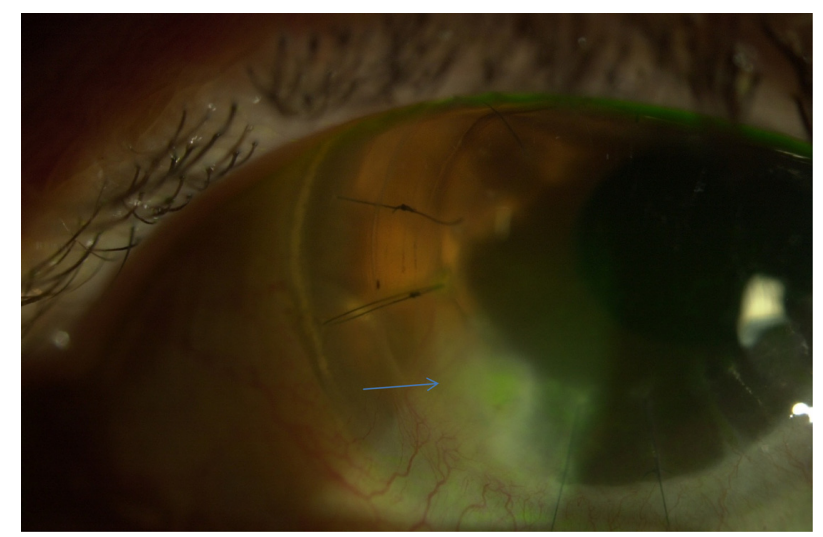

Figure $\mathbf{2}$ Slit lamp photo showing ulcer and infiltrate in corneal graft, after removal of suture (blue arrow).

A $0.5 \%$ was started four times daily for residual stromal inflammation, which was still present. One week later, there appeared to be further worsening of the infiltrate, necessitating another increase in the frequency of topical treatment. Doxycycline was also increased to $100 \mathrm{mg}$ by mouth twice a day to mitigate inflammation and treat underlying blepharitis. The patient was given nasal mupirocin two times a day for 5 days, and instructed to wash her hands and body with chlorhexidine gluconate $4 \%$ soap, with the thought of decreasing possible sources of pathogenic Staphylococci. On this regimen, the ulcer gradually healed with a stromal scar, and antibiotics were finally discontinued. The duration of treatment was 1.5 months.

\section{Discussion}

Detailed descriptions of ocular MRSA infections, specifically keratitis, have been somewhat limited in the literature. Shanmuganathan et al from the UK reported on four patients with MRSA keratitis treated with topical chloramphenicol. ${ }^{4}$ The infections were noted to be nondestructive, with only mild superficial corneal involvement. In another report of two cases, MRSA keratitis was similarly found to be mild and slowly progressive, with superficial round infiltrates. ${ }^{5}$ Topical vancomycin $2.5 \%$ was effective for therapy. Chiang and Rapuano detailed a case of MRSA keratitis that followed cataract surgery. ${ }^{6}$ Despite treatment with high-dose vancomycin, the infection recurred twice over 6 months, and ultimately required lamellar keratectomy and a conjunctival flap. Cosar et al reported on two corneal MRSA infections after cataract surgery that resolved after 60 days and 108 days, respectively. ${ }^{7}$ There have been several reports of MRSA infection after refractive surgery. ${ }^{8-12}$ Moshirfar et al presented a case of MRSA keratitis after laser in situ keratomileusis, which was resistant to fourth-generation fluoroquinolones. ${ }^{8}$
This patient required surgery to help effect a cure. Solomon et al appear to have the largest series of MRSA keratitis cases following laser refractive surgery, with their series of 13 eyes in 12 patients from multiple centers. ${ }^{9}$ They recommended initial treatment for postrefractive patients with a fourthgeneration fluoroquinolone every 30 minutes, alternating with fortified vancomycin $50 \mathrm{mg} / \mathrm{mL}$ every 30 minutes, as well as discontinuing topical steroid use. ${ }^{12}$

Our two cases of postoperative keratitis are similar to some of the foregoing postoperative reports in that the clinical presentation was severe, being associated with concurrent endophthalmitis in case 1 and keratolysis requiring corneal gluing in case 2. S. aureus in each of the cases demonstrated multiple antimicrobial resistance, including to fluoroquinolones. We have described our approach to the successful eradication of these two MRSA infections. Topical antibiotic therapy was the mainstay of treatment. Fortified vancomycin $50 \mathrm{mg} / \mathrm{mL}$ and fortified bacitracin $3000 \mathrm{U} / \mathrm{mL}$ were utilized in both cases after antimicrobial susceptibility test results were obtained. A third topical antibiotic, polymyxin B-trimethoprim, was added one month into the treatment of case 1, when the infection appeared to have flared up and worsened. Oral doxycycline was prescribed to reduce corneal collagenase activity, reduce inflammation, and treat coexistent blepharitis. Mupirocin calcium ointment was applied twice daily to the nares for 5 days, and skin antiseptics were also used, with the intention of decreasing or eliminating potential sources of MRSA.

Although our patients were not specifically tested for MRSA colonization, they were considered to have a higher risk due to their relatively compromised immune status. Type 1 diabetes mellitus is known to be a strong risk factor for MRSA colonization and infection. ${ }^{13}$ Similarly, patients with multiple myeloma have decreased immunity to $S$. aureus, and may be susceptible to MRSA. ${ }^{14,15}$ Furthermore, in case 2, the patient's previous endophthalmitis, current MRSA corneal infection, and subsequent culture positivity for coagulasenegative Staphylococcus, well into the treatment, all suggested that she might be a carrier for pathogenic bacteria, and in particular, resistant Staphylococci. ${ }^{16}$ One retrospective study at a university hospital found that up to twothirds of all patients with clinical MRSA infections had nasal colonization. ${ }^{17}$ Another study examining only MRSA skin and soft tissue infections found that $37 \%$ of patients were colonized by MRSA. ${ }^{18}$ Despite our empiric approach described above, it is probably worthwhile in general to confirm MRSA colonization and thereby guide therapy by culturing sites such as the eyelids and nares. ${ }^{9,12}$ 
Both of our MRSA cases required prolonged treatment relative to other forms of bacterial keratitis, with slow resolution only after 3 and 1.5 months, respectively. This extended clinical course was similar to the experience of some groups, but also notably longer than some other cases that have been

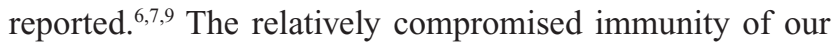
patients may have been a factor in prolonging the response to treatment. Another factor may have been the use of topical immune-modulating eyedrops. Prednisolone acetate $1 \%$ was given conservatively in order to moderate inflammation from endophthalmitis in case 1, and in case 2 to avert immunologic rejection. Topical cyclosporine A was also administered to the second patient during the latter part of her therapeutic course to counteract corneal inflammation. Without the means of direct comparison, it is unclear whether topical steroids and cyclosporine $\mathrm{A}$ in low dose have either a beneficial or detrimental influence on the clinical course. Nevertheless, certainly in case 2, it appears that use of these topical agents coincided with exacerbations of infection. Relative immune compromise likely played a role in generally increasing these patients' susceptibility to MRSA infection. Endophthalmitis additionally complicated the clinical course of both. Questions for future study may include whether immune status affects response to and duration of treatment, whether or not patients with MRSA keratitis may have greater susceptibility to endophthalmitis, and the role, if any, of topical corticosteroids.

\section{Conclusion}

The optimal approach to managing MRSA keratitis has not yet been determined. Polyantimicrobial therapy appears effective in eradicating MRSA-related postoperative keratitis. Topical fortified antibiotics, ie, vancomycin 50 $\mathrm{mg} / \mathrm{mL}$ and topical fortified bacitracin $50 \mathrm{mg} / \mathrm{mL}$, were used to treat both patients in the two case reports, with a third topical antibiotic, polymyxin B-trimethoprim, added later for the second patient. Oral doxycycline, mupirocin nasal ointment, and skin antiseptics were also used, and may be useful adjuncts in the management of this challenging condition. Full resolution of MRSA keratitis may require prolonged medical therapy in comparison with other forms of bacterial keratitis. Although the virulence of the pathogen certainly may be one factor, there may also be immunological factors at play as well. Patients in our study had underlying immunologic compromise, and were susceptible to postoperative endophthalmitis. Topical prednisolone acetate $1 \%$ and topical cyclosporine A $0.5 \%$ were also utilized to decrease inflammation during the treatment course. There is some indication that these medications may have contributed to lengthening the time needed to achieve clinical resolution. Based on this experience, we believe that topical immune-modulating medications should either not be used during the treatment of MRSA keratitis, or only used with appropriate caution.

\section{Disclosure}

The authors report no conflicts of interest in this work.

\section{References}

1. Asbell PA, Sahm DF, Shaw M, Draghi DC, Brown NP. Increasing prevalence of methicillin resistance in serious ocular infections caused by Staphylococcus aureus in the United States: 2000-2005. J Cataract Refract Surg. 2008;34:814-818.

2. Blomquist PH. Methicillin-resistant Staphylococcus aureus infections of the eye and orbit (an American Ophthalmological Society thesis). Trans Am Ophthalmol Soc. 2006;104:322-345.

3. Freidlin J, Acharya N, Lietman TM, Cevallos V, Whitcher JP, Margolis TP. Spectrum of eye disease caused by methicillin-resistant Staphylococcus aureus. Am J Ophthalmol. 2007;144:313-315.

4. Shanmuganathan VA, Armstrong M, Buller A, Tullo AB. External ocular infections due to methicillin-resistant Staphylococcus aureus (MRSA). Eye. 2005;19:284-291

5. Lee KM, Lee HS, Kim MS. Two cases of corneal ulcer due to methicillin-resistant Staphylococcus aureus in high risk groups. Korean J Ophthalmol. 2010;24:240-244.

6. Chiang RK, Rapuano CJ. Recurrent methicillin-resistant Staphylococcus aureus wound ulcer after clear-cornea cataract surgery. CLAO J. 2002;28:109-110.

7. Cosar CB, Cohen EJ, Rapuano CJ, Laibson PR. Clear corneal wound infection after phacoemulsification. Arch Ophthalmol. 2001;119: $1755-1759$.

8. Moshirfar M, Mirzaian G, Feiz V, et al. Fourth generation fluoroquinoloneresistant bacterial keratitis after refractive surgery. $J$ Cataract Refract Surg. 2006;32:515-518

9. Solomon R, Donnenfeld ED, Perry HD, et al. Methicillin-resistant Staphylococcus aureus infectious keratitis following refractive surgery. Am J Ophthalmol. 2007;143:629-634.

10. Rubinfeld RS, Negvesky GJ. Methicillin-resistant Staphylococcus aureus ulcerative keratitis after laser in situ keratomileusis. J Cataract Refract Surg. 2001;27:1523-1525.

11. Rudd JC, Moshirfar M. Methicillin-resistant Staphylococcus aureus keratitis after laser in situ keratomileusis. J Cataract Refract Surg. 2001;27:471-473.

12. Solomon R, Donnenfeld ED, Perry HD, Biser S. Bilateral methicillinresistant Staphylococcus aureus keratitis in a medical resident following an uneventful bilateral photorefractive keratectomy. Eye Contact Lens. 2003;29:187-189.

13. Kutlu SS, Cevahir N, Akalin S, et al. Prevalence and risk factors for methicillin-resistant Staphylococcus aureus colonization in a diabetic outpatient population: A prospective cohort study. Am J Infect Control. August 22, 2011. [Epub ahead of print.]

14. Karlsson J, Andréasson B, Kondori N, et al. Comparative study of immune status to infectious agents in elderly patients with multiple myeloma, Waldenstrom's macroglobulinemia, and monoclonal gammopathy of undetermined significance. Clin Vaccine Immunol. 2011;18: 969-977.

15. Sakai C, Satoh Y, Ohkusu K, Kumagai K, Ishii A. Outbreak of methicillin-resistant Staphylococcus aureus (MRSA) infection or colonization among patients with neoplastic disease: a clinic-epidemiological study of 11 cases. Kansenshogaku Zasshi. 2001;75:940-945. Japanese. 
16. Pinna A, Zanetti S, Sotglu M, Sechi LA, Fadda G, Carta F. Identification and antibiotic susceptibility of coagulase negative staphylococci isolated in corneal/external infections. Br J Ophthalmol. 1999;83:771-773.

17. Robicsek A, Suseno M, Beaumont JL, Thomson RB Jr, Peterson LR. Prediction of methicillin-resistant Staphylococcus aureus involvement in disease sites by concomitant nasal sampling. J Clin Microbiol. 2008;46:588-592.
18. Yang ES, Tan J, Eells S, Rieg G, Tagudar G, Miller LG. Body site colonization in patients with community-associated methicillin-resistant Staphylococcus aureus and other types of S. aureus skin infections. Clin Microbiol Infect. 2010;16:425-431.

\section{Publish your work in this journal}

Clinical Ophthalmology is an international, peer-reviewed journal covering all subspecialties within ophthalmology. Key topics include: Optometry; Visual science; Pharmacology and drug therapy in eye diseases; Basic Sciences; Primary and Secondary eye care; Patien Safety and Quality of Care Improvements. This journal is indexed on

Submit your manuscript here: http://www.dovepress.com/clinical-ophthalmology-journal

\section{Dovepress}

PubMed Central and CAS, and is the official journal of The Society of Clinical Ophthalmology (SCO). The manuscript management system is completely online and includes a very quick and fair peer-review system, which is all easy to use. Visit http://www.dovepress.com/ testimonials.php to read real quotes from published authors. 